Check for updates

Cite this: J. Mater. Chem. A, 2021, 9, 19675

Received 20th March 2021 Accepted 1st June 2021

DOI: $10.1039 / \mathrm{d} 1 \mathrm{ta0} 02351 \mathrm{a}$

rsc.li/materials-a

\section{Dopants modulate crystal growth in molten salts enabled by surface energy tuning $\dagger$}

\author{
Xiaoqiao Li, ț ${ }^{\text {ab }}$ Linming Zhou, (D) $!^{\mathrm{c}}$ Han Wang, ${ }^{\mathrm{b}}$ Dechao Meng, ${ }^{\mathrm{b}}$ Guannan Qian, ${ }^{\mathrm{b}}$ \\ Yong Wang, ${ }^{\mathrm{b}}$ Yushi He, (D) ${ }^{\mathrm{b}}$ Yongjun Wu, (D) ${ }^{c}$ Zijian Hong, (D) ${ }^{* c}$ Zi-Feng Ma (D) ${ }^{b}$ \\ and Linsen $\mathrm{Li}(\mathbb{D}$ *bd
}

Crystalline materials are routinely produced via high-temperature synthesis and show size-dependent properties; however, a rational approach to regulating their crystal growth has not been established. Here we show that dopants traditionally used for modifying crystal lattices can also function as growth mediators in molten-salt synthesis by altering the surface energy and thus the nucleation barrier and the critical nuclei size. This was demonstrated by size-tunable synthesis of lithium cobalt oxide $\left(\mathrm{LiCoO}_{2}\right)$ with a trace amount of titanium oxide or tungsten oxide as the dopant. The understanding of the dopantmediated growth mechanism allows the rational design and control of the particle size and the doping for $\mathrm{LiCOO}_{2}$, enabling a high energydensity battery cathode with exceptional rate capability and cycle stability at high voltages intolerable for conventional $\mathrm{LiCoO}_{2}$.

\section{Introduction}

Crystal growth is a fundamental process and a key step in a wide range of applications including microelectronics, pharmaceutics, catalysis, and energy storage. Tailoring the size of individual crystals is often essential from the perspectives of performance and/or cost. ${ }^{\mathbf{1 - 3}}$ For instance, increasingly larger silicon ingots are grown via the Czochralski method by controlling the temperature and pulling rate of the seed crystal so that wafers with larger diameters (now at $450 \mathrm{~mm}$ ) can be made to enhance productivity and efficiency in semiconductor device fabrication. Nanocrystal-based or even single-atom

${ }^{a}$ College of Environmental \& Chemical Engineering, Shanghai University of Electric Power, Shanghai 200090, China

${ }^{b}$ Department of Chemical Engineering, Shanghai Jiao Tong University, Shanghai 200240, China. E-mail: linsenli@sjtu.edu.cn

${ }^{c}$ School of Materials Science \& Engineering, Zhejiang University, Hangzhou 310027, China. E-mail: hongzijian10o@zju.edu.cn

${ }^{d}$ Shanghai Jiao Tong University, Sichuan Research Institute, Chengdu 610213, China $\dagger$ Electronic supplementary information (ESI) available. See DOI: 10.1039/d1ta02351a

$\ddagger$ These authors contributed equally to this work. catalysts are synthesized by colloidal chemistry or thermal pyrolysis to maximize the surface-to-volume ratio and catalytic efficiency. ${ }^{2,3}$ Electrode materials for batteries store alkali ions (e.g. $\mathrm{Li}^{+}, \mathrm{Na}^{+}$, and $\mathrm{K}^{+}$) at interstitial sites in the bulk and their electrochemical performance is highly dependent on the particle size. ${ }^{4-6}$ Larger particles are favorable for achieving higher packing density and volumetric energy density but there are size thresholds above which the power capability is compromised. Taking layered-oxide materials $\left(\mathrm{LiMO}_{2}, \mathrm{M}=\mathrm{Co}\right.$, $\mathrm{Ni}-\mathrm{Mn}-\mathrm{Co}$, or Ni-Co-Al) as examples, which are widely used in consumer electronics and electric vehicles, we may estimate the optimal grain size $\left[L=2(D t)^{0.5}\right]$ based on their typical Li diffusivity $\left(D=10^{-10}-10^{-12} \mathrm{~cm} \mathrm{~s}^{-1}\right)$ and the assumption that the electrochemical reaction is limited by bulk diffusion., ${ }^{4,7}$ The grain size should be in the range of $10^{\circ}-10^{1} \mu \mathrm{m}$ in order to fully utilize the capacity at practically relevant $\mathrm{C}$-rates $(0.2$ to $2 \mathrm{C}$, i.e. 5 to $0.5 \mathrm{~h}$ for one charge or discharge). These oxide materials are currently prepared via high-temperature solid-state reactions that have little control over the size of the individual particles and require pulverization and sieving to achieve a desirable particle size distribution. Although rational control of crystal growth has been made possible with the assistance of surfactants in low-temperature solution synthesis ${ }^{3}$ or enabled by supersaturation control, ${ }^{8}$ it remains a challenge to do the same in high-temperature synthesis.

The molten-salt-assisted approach significantly enhances ion diffusion and reaction kinetics by using a molten salt as the "liquid phase" reaction medium. ${ }^{9-12}$ It offers better control over the size, elemental composition, and crystal shape of metal oxide materials but generally requires stringent control over a set of experimental variables, such as the composition of salts, salt-to-reactant ratios, reaction temperature, time, nature of metal precursors, etc. Here we present direct observation and mechanistic investigation of crystal growth regulation via a single experimental variable, using the molten-salt synthesis of $\mathrm{LiCoO}_{2}$ (LCO) as a model system. LCO is a technologically important battery material widely used in consumer electronics. ${ }^{13}$ We discovered that dopants traditionally used for 
modifying the lattice structure and improving the electrochemical performance of LCO can also act as growth mediators in the synthesis by altering the surface energy and thus the nucleation barrier and the critical nuclei size. Simultaneous control of the grain size and the structure of LCO leads to a high energy-density battery cathode with exceptional rate capability and cycle performance.

\section{Results and discussion}

Doping is a technological process widely adopted in materials science and involves incorporating foreign atoms or ions into host lattices to achieve desirable properties and functions. LCO has been doped with numerous foreign elements to improve its cycle stability, especially when it is charged to high voltages $(\geq 4.5 \mathrm{~V})$ to increase the accessible energy density. ${ }^{13-15}$ However, little attention has been paid to the dopants' role in the crystal growth. Previous studies have revealed significantly different solubility of dopants in the LCO lattice. For example, $\mathrm{Mg}$ and $\mathrm{Al}$ show a solubility of $0.5 \mathrm{~mol} \%$ or higher at Co sites whereas $\mathrm{Zr}$ is less soluble and $\mathrm{Ti}$ is known to segregate on the LCO particle surface or at grain boundaries (i.e. barely soluble). ${ }^{15-17}$ Since many metal oxides are known to dissolve in common molten salts, ${ }^{18}$ we reasoned that they should interact with the growing surface and thus modify the formation process of the LCO grains.

To valid our hypothesis, $\mathrm{Mg}^{2+}, \mathrm{Al}^{3+}, \mathrm{Ti}^{4+}, \mathrm{Zr}^{4+}, \mathrm{Nb}^{5+}, \mathrm{Mo}^{6+}$, and $\mathrm{W}^{6+}$ ions were introduced into the synthesis of LCO in $\mathrm{LiOH}-\mathrm{Li}_{2} \mathrm{SO}_{4}$ molten salts at $910{ }^{\circ} \mathrm{C}$ in air at the same molar fraction ( $1 \mathrm{~mol} \%$ relative to $\mathrm{Co}$ and $0.5 \mathrm{~mol} \%$ relative to the molten salts; see the Methods in the ESI $\dagger)$. Cobalt oxide $\left(\mathrm{Co}_{3} \mathrm{O}_{4}\right)$ was used as the Co source. After the synthesis, the LCO particles were isolated from the salts by water washing. All the samples were first examined by powder X-ray diffraction (PXRD) and were indexed to the hexagonal $\alpha-\mathrm{NaFeO}_{2}$-type structure without any detectable impurity phases (ESI Fig. $1 \dagger$ ). Scanning electron microscopy (SEM) characterization was performed to evaluate the impact of different dopants on the crystal growth of LCO (Fig. 1). Without the dopants added, the pure LCO particles grew up to approximately $30 \mu \mathrm{m}$ in size (Fig. 1a). The addition of $\mathrm{Mg}^{2+}$ and $\mathrm{Al}^{3+}$ had little impact on the particle size (Fig. $1 \mathrm{~b}$ and c), whereas the size reduction was evident in all other cases. In particular, $\mathrm{Ti}^{4+}$ and $\mathrm{W}^{6+}$ doping reduced the LCO particle size by almost one order of magnitude (from $\sim 30 \mu \mathrm{m}$ to $\sim 2-3 \mu \mathrm{m}$, Fig. 1d and h). More structural characterization results can be found in ESI Fig. 2. $\dagger$

To shed more light on the effect of dopant ions in mediating the crystal growth, we performed first-principles calculations based on density functional theory (DFT, see details in the ESI $\dagger$ ) and evaluated the surface energy change of the LCO surface due to the cation doping (Fig. 2). Two types of low-index, low-energy LCO surfaces were modeled, namely the (0001) and (1014) surfaces. ${ }^{19}$ The crystal structures of the undoped and cation doped (0001) and (1014) surface slabs are plotted in Fig. 2a-d. Two Co ions on the top and bottom of the slab are replaced by the dopants, as shown in Fig. $2 \mathrm{~b}$ and d. In order to minimize the charging effect, a thick (0001) surface was built with 6 Li layers. The surface energy was calculated by taking the difference of the total energy of the slab and the bulk, i.e. $\gamma=\frac{1}{2 A}\left(G_{\text {slab }}-G_{\text {bulk }}\right)$.The surface energies of undoped LCO (0001) and (1014) surfaces were calculated to be $2.181 \mathrm{~J} \mathrm{~m}^{-2}$ and $1.111 \mathrm{~J} \mathrm{~m}^{-2}$, respectively, which agreed well with a previous report. ${ }^{19}$ We then moved on to understand the impact of the dopants on the surface energy, including $\mathrm{Mg}, \mathrm{Al}, \mathrm{Ti}, \mathrm{Zr}, \mathrm{Nb}, \mathrm{Mo}$, and $\mathrm{W}$. The results are summarized in ESI Table $1 . \dagger$ We plot the surface energy reduction in Fig. 2e, which is calculated by extracting the energy difference for the doped and undoped surfaces. It can be observed that the substitution of Co by $\mathrm{W}$ can
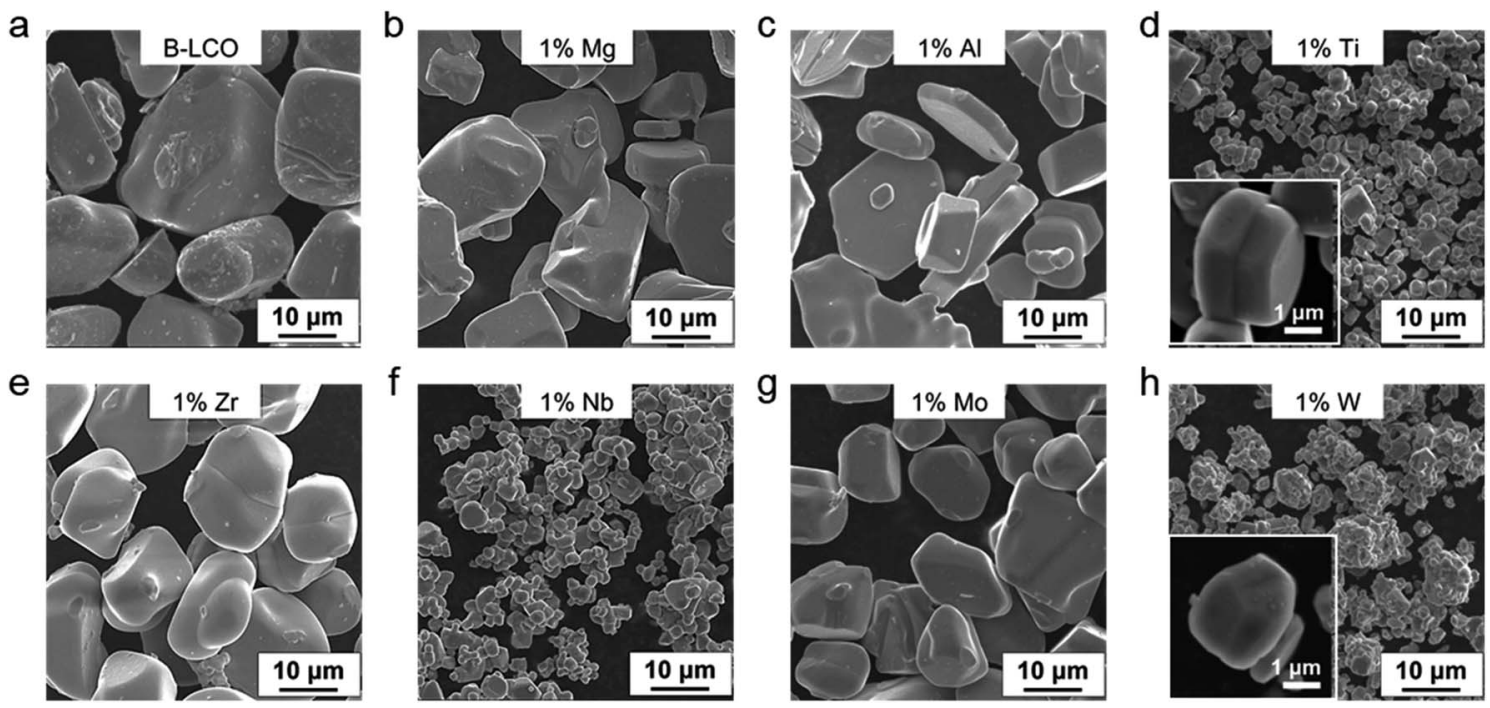

Fig. 1 SEM micrographs of LCO particles. (a) LCO particles grown without dopants via the molten-salt synthesis. (b)-(h) Particles grown under identical conditions except for the addition of $1 \mathrm{~mol} \%$ of $\mathrm{Mg}^{2+}, \mathrm{Al}^{3+}, \mathrm{Ti}^{4+}, \mathrm{Zr}^{4+}, \mathrm{Nb}^{5+}, \mathrm{Mo}^{6+}$, and $\mathrm{W}^{6+}$ (relative to Co), respectively. Size reduction is evident for $\mathrm{Ti}^{4+}, \mathrm{Zr}^{4+}, \mathrm{Nb}^{5+}, \mathrm{Mo}^{6+}$, and $\mathrm{W}^{6+}$ but not for $\mathrm{Mg}^{2+}$ and $\mathrm{Al}^{3+}$. 
a

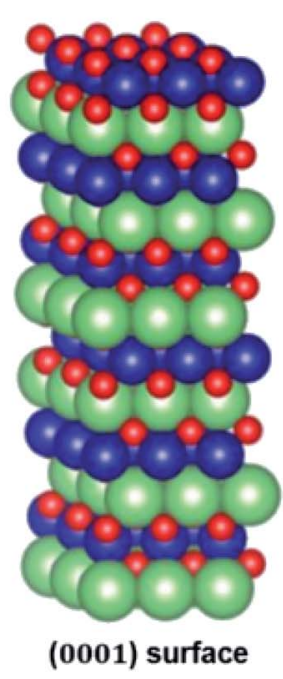

b

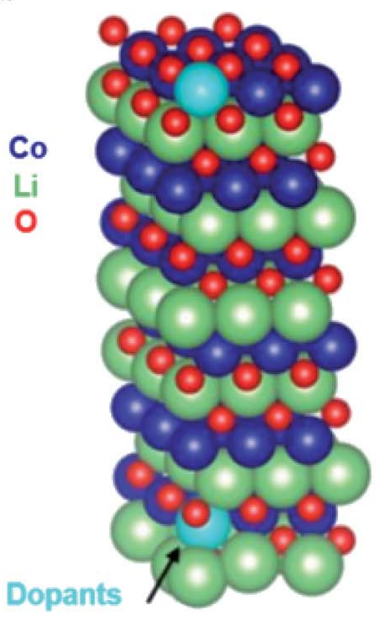

C

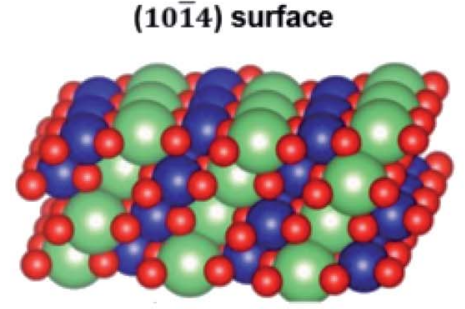

d

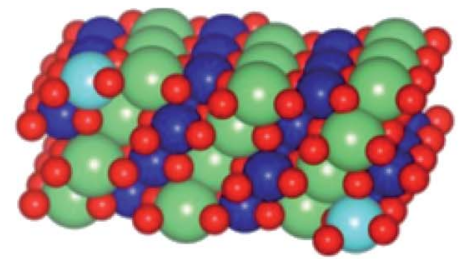

e

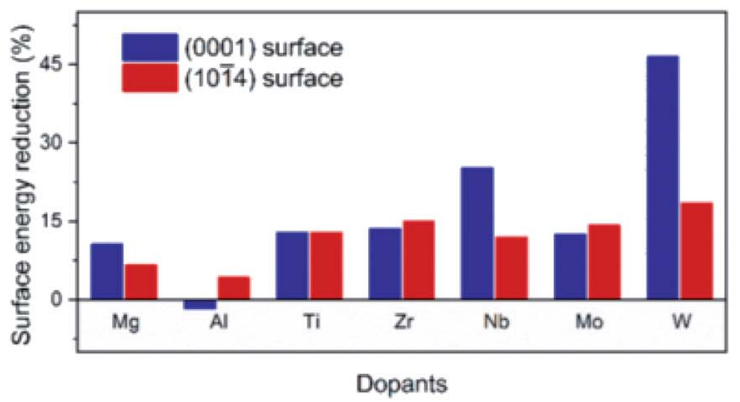

f

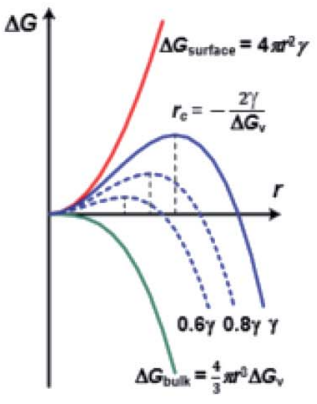

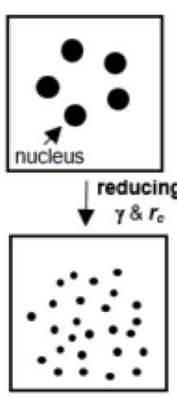

Fig. 2 Surface energy tuning by the dopants predicted by DFT calculations. (a)-(d) The crystal structures of the undoped and doped (0001) and (1014) surface slabs of LCO, respectively. (e) Bar plots showing the percentage of surface energy reduction induced by the dopants for the lowindex, low-energy LCO surfaces we selected. Doping with W, Nb, Ti, Zr, or Mo leads to a more significant reduction in surface energy than Mg and Al ions, which strongly impacts the nucleation processes according to classical nucleation theory as schematically shown in (f). The critical nucleus size $r_{\mathrm{c}}$ is proportional to the surface energy $\gamma\left(r_{\mathrm{c}}=-\frac{2 \gamma}{\Delta G_{\mathrm{v}}}\right)$, because the change in the Gibbs free energy per volume due to the phase transition $\left(\Delta G_{v}\right)$ is small with no more than $1 \mathrm{~mol} \%$ of the dopants incorporated into the LCO lattice. Reducing the surface energy $\gamma$ leads to smaller $r_{\mathrm{c}}$, which promotes the formation of more and smaller nuclei.

greatly change the surface energy of LCO, leading to $\sim 46 \%$ and $\sim 18 \%$ reduction of $(0001)$ and (1014) surface energies, respectively (Fig. 2e). Doping with $\mathrm{Nb}, \mathrm{Ti}, \mathrm{Zr}$ and Mo also shows a significant reduction in the surface energies, whereas $\mathrm{Mg}$ and $\mathrm{Al}$ ion substitutions have relatively weaker effects.

From classical nucleation theory (schematically shown in Fig. 2f), one can write: $\Delta G=\frac{4}{3} \pi r^{3} \Delta G_{v}+4 \pi r^{2} \gamma$, where $\Delta G$ is the Gibbs free energy change due to the nucleation, $\Delta G_{\mathrm{v}}$ is the Gibbs free energy per volume due to the phase transition, $r$ is the radius of the nuclei, and $\gamma$ is the surface energy ${ }^{20}$ The minimization of $\Delta G$ with respect to $r$ gives: $r_{\mathrm{c}}=-\frac{2 \gamma}{\Delta G_{\mathrm{v}}}$, where $r_{\mathrm{c}}$ is the critical nucleus size. Since the doping level in this study is no more than $1 \%$, the change in $\Delta G_{\mathrm{v}}$ is considerably small for different dopants, and $r_{\mathrm{c}}$ is thus directly proportional to the surface energy $\gamma$. Reducing the surface energy could lead to smaller nuclei, which subsequently grow into smaller crystals because the total amount of the reactants is a constant in a batch synthesis. Moreover, the nucleation barrier is proportional to $\gamma^{3}$, and reducing the surface energy could lead to a large reduction in the nucleation barrier which leads to more nuclei in the system. It can be theoretically expected that the doping with $\mathrm{W}, \mathrm{Ti}, \mathrm{Nb}, \mathrm{Mo}$ and $\mathrm{Zr}$ will reduce the grain size of LCO whereas $\mathrm{Al}$ and $\mathrm{Mg}$ show minimal effect, which is consistent with our experimental observations (Fig. 1). However, we do want to point out that the dopant-induced surface energy reduction effect may not be the sole reason for the reduction of the LCO particle size. Besides the nucleation process, the dopants could also alter the particle growth process, which may explain the individual dopants' deviations from the overall trend as predicted by the DFT calculations.

With the understanding of the dopant's role in the synthesis, we can rationalize the use of $\mathrm{Ti}^{4+}$ or $\mathrm{W}^{6+}$ not just as a dopant but also a mediator for the crystal growth of LCO at high temperature, similar to the role of surfactants or ligands in the lowtemperature solution synthesis. Indeed, the size of the LCO particles could be continuously tuned by varying the $\mathrm{Ti}^{4+}$ or $\mathrm{W}^{6+}$ concentration in the molten salts. The primary particle size decreased from $\sim 15 \mu \mathrm{m}$ to $\sim 2 \mu \mathrm{m}$ as the $\mathrm{Ti}^{4+}$ or $\mathrm{W}^{6+}$ 
a

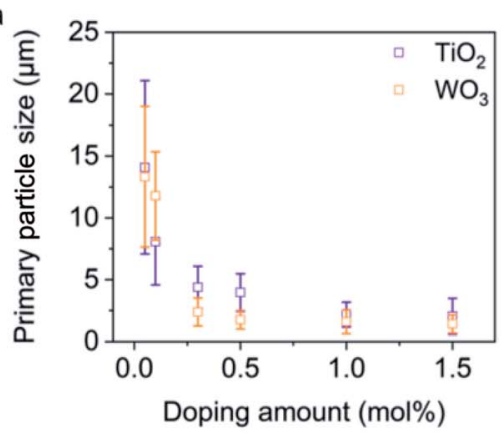

b

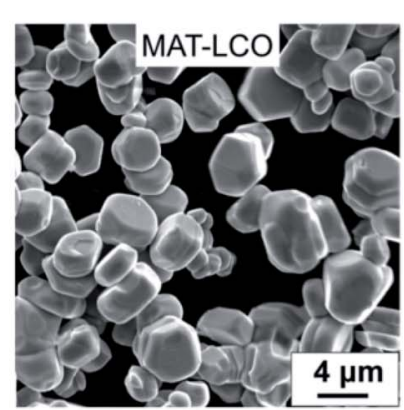

c

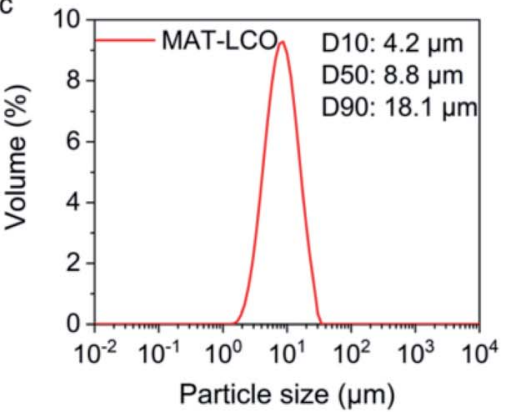

d

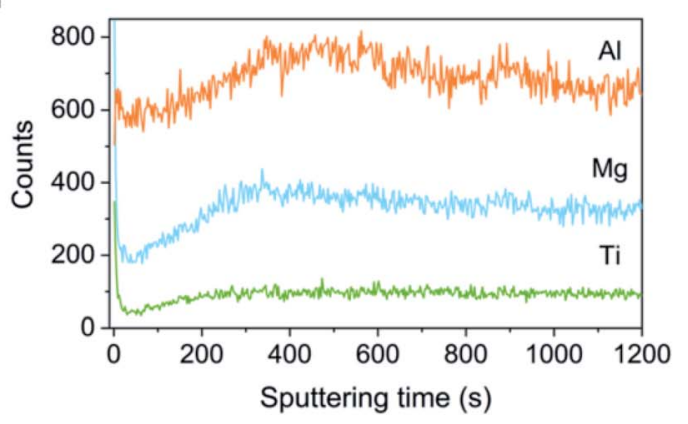

e
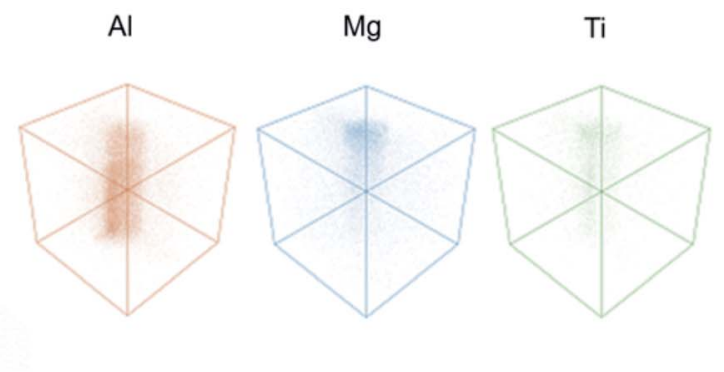

Fig. 3 Rational design and synthesis of LCO using selected dopants as the growth mediator. (a) Average primary particle size of the LCO particles as a function of the dopant $\left(\mathrm{Ti}^{4+}\right.$ and $\left.\mathrm{W}^{6+}\right)$ concentration in the molten-salt synthesis. (b) $\mathrm{LCO}$ particles synthesized with $\mathrm{Mg}^{2+}, \mathrm{Al}^{3+}$, and $\mathrm{Ti}^{4+}$ as co-dopants (MAT-LCO). $\mathrm{Ti}^{4+}$ ions also act as the mediator for crystal growth to control the grain size. (c) Particle size distribution of the MATLCO particles. (d) and (e) Depth profiling and three dimensional elemental mapping results obtained by TOF-SIMS, showing the spatial distribution of the $\mathrm{Mg}^{2+}, \mathrm{Al}^{3+}$, and $\mathrm{Ti}^{4+}$ dopants in MAT-LCO particles. The sputtered volume is $10 \mu \mathrm{m} \times 10 \mu \mathrm{m} \times 1 \mu \mathrm{m}$.

concentration increased from $0.01 \mathrm{~mol} \%$ to $1.5 \mathrm{~mol} \%$ (Fig. 3a, SEM images in ESI Fig. $3 \dagger)$. This dopant-mediated crystal growth approach allows us to target a certain particle size and easily achieve it by tuning a single variable (dopant concentration), which is in stark contrast to the conventional synthetic methods that often involve adjusting several variables and a large number of trial-and-error investigations. For example, we deterministically prepared a LCO sample (denoted as MATLCO) with a median size $\left(D_{50}\right)$ of $\sim 8.8 \mu \mathrm{m}$ and achieved high homogeneity in the particle size and morphology (Fig. 3b and c) by using $\sim 0.3 \mathrm{~mol} \%$ of $\mathrm{Ti}^{4+}$ as the mediator for crystal growth in the molten salts and having $\mathrm{Mg}^{2+}(0.5 \mathrm{~mol} \%), \mathrm{Al}^{3+}(0.5 \mathrm{~mol} \%)$ ions as co-dopants (in addition to the $\mathrm{Ti}^{4+}$ ions). This could also be done by using $\mathrm{W}^{6+}$ as the growth mediator (ESI Fig. $4 \dagger$ ). As we showed earlier, $\mathrm{Mg}^{2+}$ and $\mathrm{Al}^{3+}$ ions had a minimal impact on the LCO growth. They were reported to improve structural stability during deep delithiation ${ }^{\mathbf{1 3}}$ and thus introduced. PXRD and Rietveld refinements confirmed that MAT-LCO had a highly ordered layered structure without detectable impurity phases (ESI Fig. $5 \dagger$ ). Time-of-flight secondary-ion mass-spectrometry (TOF-SIMS) characterization was performed to evaluate the spatial distribution of the dopants. Depth profiling (Fig. 3d) and three-dimensional elemental mapping (Fig. 3e) show that the $\mathrm{Al}^{3+}$ ions are uniformly dispersed in the LCO particle whereas a relatively higher concentration of $\mathrm{Mg}^{2+}$ and $\mathrm{Ti}^{4+}$ ions is located near the surface.

The rationally designed MAT-LCO cathode material enabled excellent electrochemical performance at a high cut-off voltage of $4.5 \mathrm{~V}$ (versus $\mathrm{Li} / \mathrm{Li}^{+}$). It displayed an exceptionally high capacity of $193.6 \mathrm{~mA} \mathrm{~h} \mathrm{~g}^{-1}$ and retained more than $97 \%$ of the initial capacity over 100 cycles at $0.33 \mathrm{C}$ (Fig. 4a). In comparison, the undoped LCO (B-LCO) was only stable at a low cut-off voltage of $4.2 \mathrm{~V}$ but rapidly degraded when it was cycled to $4.5 \mathrm{~V}$ and lost more than $20 \%$ of the initial capacity after 100 cycles. According to the literature, the rapid performance decay of the undoped LCO during high-voltage cycling could be caused by both bulk and surface instabilities, including harmful phase transitions (O3 to $\mathrm{H} 1-3$ or even $\mathrm{O} 1$ ), microcrack formation, oxygen loss, and electrolyte decomposition. ${ }^{13}$ A further inspection of the voltage curves in the $4^{\text {th }}$ and $107^{\text {th }}$ cycles revealed a large increase in cell polarization and significant capacity loss for B-LCO (Fig. 4b) but not for MAT-LCO (Fig. 4c), proving its excellent interfacial and lattice stabilities enabled by the structural doping. Furthermore, MAT-LCO exhibited outstanding rate capability unmatched by B-LCO and delivered more than $170 \mathrm{~mA} \mathrm{~h} \mathrm{~g}^{-1}$ when it was charged and discharged at an extreme high rate of 20C (i.e. 3 min charge-discharge, Fig. 4d). The Ragone plot in Fig. 4e compares the electrochemical performance of MAT-LCO and bare LCO in terms of energy density and power density, proving that MAT-LCO is a high-energy and high-power battery cathode material. We further compared the long-term cycle performance of MATLCO with that of commercial LCO (c-LCO) in full cells using artificial graphite as the anode. c-LCO is the same material now widely used in high-end consumer electronics and designed for $4.35 \mathrm{~V}$ LCO-graphite full batteries. In our tests, 

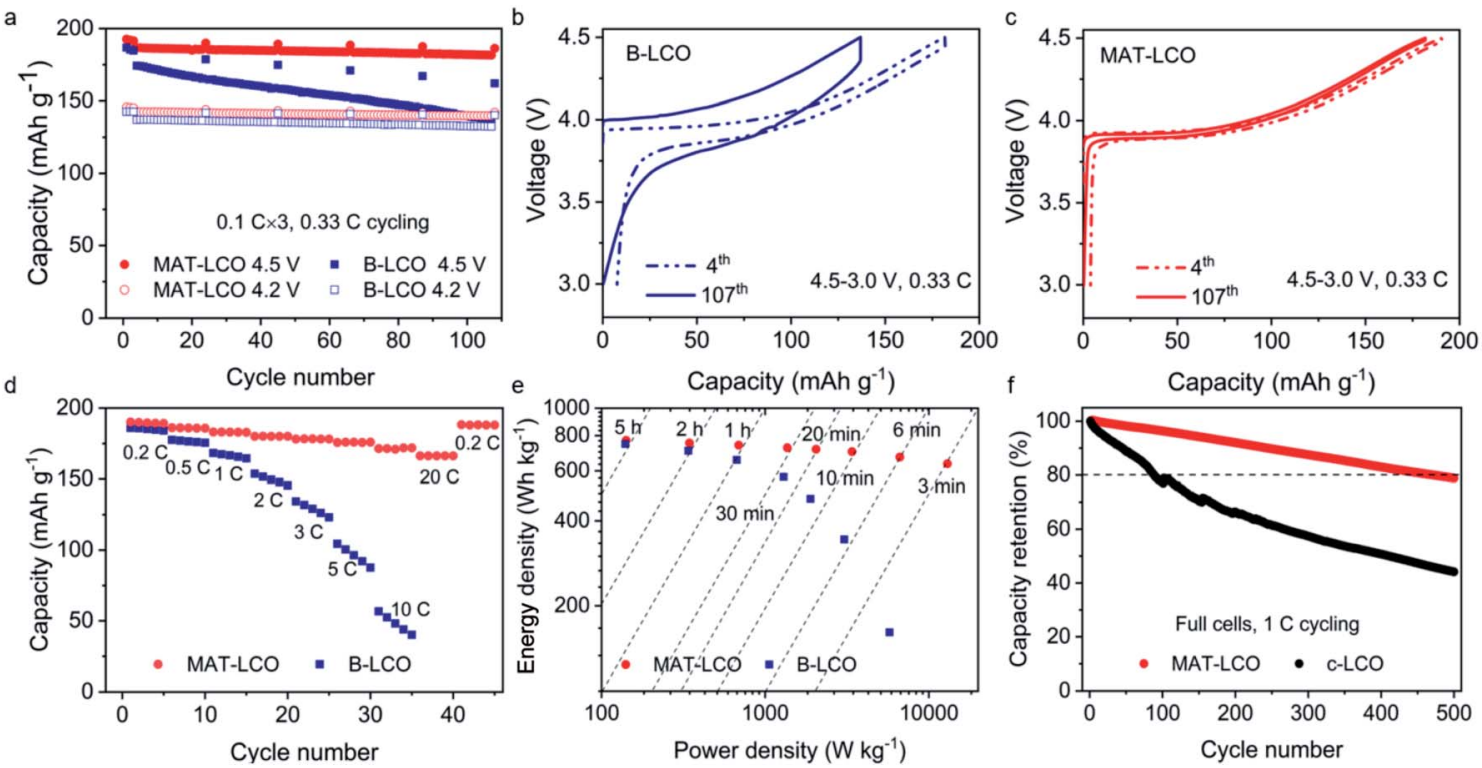

Fig. 4 Electrochemical characterization of $\mathrm{LiCoO}_{2}$ cathodes. (a) Cycle performance of the LCO|Li half cells. The cells went through three formation cycles at $0.1 \mathrm{C}$ before being cycled at $0.33 \mathrm{C}$. (b) and (c) Voltage profiles of B-LCO and MAT-LCO for the $4^{\text {th }}$ and $107^{\text {th }}$ cycles at $0.33 \mathrm{C}$. (d) Rate performance. The cells were charged and discharged at equivalent C-rates in each cycle. (e) Ragone plot of the LCO/Li cells. (f) Longterm full-cell cycling tests of the MAT-LCO cathodes using artificial graphite as the anode in comparison with commercial LCO currently used in consumer electronics.

the full cells were charged to $4.4 \mathrm{~V}$ to evaluate the high-voltage cycle stability. The full cell with MAT-LCO retained $\sim 80 \%$ of the initial capacity over 500 cycles, whereas c-LCO was clearly not suitable for high-voltage cycling, as evidenced by its rapid capacity decay (Fig. 4e) and much lower CEs $(<99 \%$, ESI Fig. $6 \dagger$ ).

In conclusion, we have established a theoretical framework for regulating the crystal growth in molten salts via dopantinduced surface energy tuning and experimentally demonstrated the rational synthesis of LCO cathode materials with desirable particle size, doping, and exceptional electrochemical performance under harsh conditions. These findings enrich our understanding of crystal growth at high temperature and enable tremendous improvements in our ability to design and synthesize functional materials. We suggest that the dopantmediated crystal growth mechanism proposed for LCO is likely general to many other materials commonly prepared via molten-salt synthesis, such as ceramics ${ }^{9}$ and complex oxides ${ }^{\mathbf{1 0 , 1 1}}$ including superconductors. ${ }^{21}$

\section{Author contributions}

Conceptualization: LSL and ZH. Investigation: XL, LZ, HW, DM, GQ, YW, YH, and YW. Visualization: XL, LSL, and ZH. Funding acquisition: LSL, ZFM, and ZH. Supervision: LSL, ZFM, and ZH. Writing - original draft: LSL and HZ. Writing - review \& editing: LSL, HZ, and MZF.

\section{Conflicts of interest}

The authors declare that they have no competing interests.

\section{Acknowledgements}

This work is supported by the Natural Science Foundation of China (22008154 to LSL), and partially by the Sichuan Science and Technology Program (2021JDRC0015 to LSL) and Sinopec (420038-1 to LSL). Z. H. would like to acknowledge a start-up grant from Zhejiang University. The DFT calculations were performed at the Shanghai Supercomputing Center, on the Mofang III cluster.

\section{Notes and references}

1 S. W. Boettcher, J. M. Spurgeon, M. C. Putnam, E. L. Warren, D. B. Turner-Evans, M. D. Kelzenberg, J. R. Maiolo, H. A. Atwater and N. S. Lewis, Science, 2010, 327, 185-187.

2 B. Qiao, A. Wang, X. Yang, L. F. Allard, Z. Jiang, Y. Cui, J. Liu, J. Li and T. Zhang, Nat. Chem., 2011, 3, 634-641.

3 V. F. Puntes, K. M. Krishnan and A. P. Alivisatos, Science, 2001, 291, 2115-2117.

4 A. Van der Ven, J. Bhattacharya and A. A. Belak, Acc. Chem. Res., 2013, 46, 1216-1225.

5 M. D. Radin, S. Hy, M. Sina, C. Fang, H. Liu, J. Vinckeviciute, M. Zhang, M. S. Whittingham, Y. S. Meng and A. Van der Ven, Adv. Energy Mater., 2017, 7, 1602888.

6 K. J. Griffith, K. M. Wiaderek, G. Cibin, L. E. Marbella and C. P. Grey, Nature, 2018, 559, 556-563.

7 M. S. Whittingham, Chem. Rev., 2004, 104, 4271-4302.

8 S. A. Morin, M. J. Bierman, J. Tong and S. Jin, Science, 2010, 328, 476-480.

9 T. Kimura, in Advances in Ceramics - Synthesis and Characterization, Processing and Specific Applications, ed. C. Sikalidis, IntechOpen, 2010. 
10 D. E. Bugaris and H.-C. zur Loye, Angew. Chem., Int. Ed., 2012, 51, 3780-3811.

11 S. Flynn, S. Sanghvi, M. L. Nisbet, K. J. Griffith, W. Zhang, P. S. Halasyamani, S. M. Haile and K. R. Poeppelmeier, Chem. Mater., 2020, 32, 4785-4794.

12 A. Dash, R. Vaßen, O. Guillon and J. Gonzalez-Julian, Nat. Mater., 2019, 18, 465-470.

13 Y. Lyu, X. Wu, K. Wang, Z. Feng, T. Cheng, Y. Liu, M. Wang, R. Chen, L. Xu, J. Zhou, Y. Lu and B. Guo, Adv. Energy Mater., 2021, 11, 2000982.

14 Q. Liu, X. Su, D. Lei, Y. Qin, J. Wen, F. Guo, Y. A. Wu, Y. Rong, R. Kou, X. Xiao, F. Aguesse, J. Bareño, Y. Ren, W. Lu and Y. Li, Nat. Energy, 2018, 3, 936-943.

15 J.-N. Zhang, Q. Li, C. Ouyang, X. Yu, M. Ge, X. Huang, E. Hu, C. Ma, S. Li, R. Xiao, W. Yang, Y. Chu, Y. Liu, H. Yu,
X.-Q. Yang, X. Huang, L. Chen and H. Li, Nat. Energy, 2019, 4, 594-603.

16 Y. Koyama, H. Arai, I. Tanaka, Y. Uchimoto and Z. Ogumi, J. Mater. Chem. A, 2014, 2, 11235-11245.

17 S. Kim, S. Choi, K. Lee, G. J. Yang, S. S. Lee and Y. Kim, Phys. Chem. Chem. Phys., 2017, 19, 4104-4113.

18 K. Sridharan and T. R. Allen, in Molten Salts Chemistry, ed. F. Lantelme and H. Groult, Elsevier, Oxford, 2013, pp. 241-267.

19 D. Kramer and G. Ceder, Chem. Mater., 2009, 21, 3799-3809. 20 W. K. Burton, N. Cabrera and F. C. Frank, Philos. Trans. R. Soc., A, 1951, 243, 299-358.

21 W. K. Ham, G. F. Holland and A. M. Stacy, J. Am. Chem. Soc., 1988, 110, 5214-5215. 